\title{
Multi-PMT optical modules for IceCube-Gen2
}

\author{
The IceCube-Gen2 Collaboration ${ }^{1}$, \\ ${ }^{1}$ http://icecube.wisc.edu/collaboration/authors/icrc15_gen2 \\ E-mail: lew.classen@physik.uni-erlangen.de
}

Following the first observation of astrophysical high-energy neutrinos by IceCube, planning for a next-generation neutrino detector at the South Pole is under way, which will significantly expand IceCube's sensitivity both towards high and low neutrino energies. In addition to upgrading the proven IceCube design, new optical sensor concepts are explored which have the potential to substantially enhance the performance of IceCube-Gen2. One concept is the multi-PMT optical module which, in contrast to the "conventional" layout with a single 10-inch photomultiplier (PMT), features 24 3-inch PMTs inside a pressure vessel. This design results in several advantages such as increased effective area, improved angular acceptance and better directional sensitivity. The layout is based on the proven design of the KM3NeT optical module which is now being adapted and enhanced for the use in the deep ice. We present the current state of the hardware developments as well as first simulations investigating the impact of multi-PMT modules on detector performance.

Corresponding authors: Lew Classen ${ }^{* 1}$, Alexander Kappes ${ }^{1}$,

${ }^{1}$ ECAP, University of Erlangen-Nuremberg, Erlangen, Germany

The 34th International Cosmic Ray Conference,

30 July- 6 August, 2015

The Hague, The Netherlands

*Speaker. 


\section{Multi-PMT concept}

Currently operating large volume neutrino telescopes, namely IceCube [1], ANTARES [2] and BAIKAL [3], are composed of optical modules with a single large photomultiplier tube (PMT). The IceCube DOM, for instance, consists of a 13-inch glass sphere housing a 10-inch Hamamatsu R7081-2 PMT [4] a $\mu$-metal cage and digitization electronics [5]. In contrast, a multi-PMT optical module, first developed for the KM3NeT neutrino telescope, houses an array of several small PMTs and their read-out electronics [6]. One of the main drivers for the development was the reduction of the number of costly and risky underwater connectors present in the ANTARES design. However, the approach has a number of other attractive advantages compared to the conventional design at a similar overall price per photocathode area:

- Increase of photocathode area: The photocathode area of ten 3-inch PMTs roughly corresponds to that of one 10-inch tube. Thus a multi-PMT DOM containing several tens of 3-inch PMTs is equivalent to several conventional IceCube optical modules.

- Superior photoelectron counting: The number of photoelectrons can be more directly derived from the number of hit PMTs compared to the extraction from a multi-photoelectron signal waveform.

- Improved angular acceptance: With the distribution of the photocathode area across the total solid angle, a near homogeneous $4 \pi$ coverage can be achieved (see Fig. 3).

- Directional sensitivity: The orientation of the hit PMT carries information on the direction of the incoming photon that can be used in the reconstruction.

The hardware functionality and physics capability of the multi-PMT concept have been demonstrated in-situ with the deployment and operation of KM3NeT prototypes in the Mediterranean [7]. As its properties give the multi-PMT module the potential to significantly enhance the performance of future neutrino detectors, it is, together with the WOM [8] and the D-Egg [9], one of the new optical sensor concepts under investigation for the IceCube-Gen2 project [10].

\section{Prototype development for IceCube-Gen2: The mDOM}

\subsection{Hardware design}

The adaptation of the multi-PMT layout for a deep-ice environment benefits from the research and development made for KM3NeT, in particular from the availability of suitable three-inch PMTs and corresponding low-power high voltage circuitry. However, the use in deep ice requires a major redesign of the pressure vessel. The constraints of using the currently available drilling technology and avoiding significant increase of costs limit the module diameter to approximately 14 inches $(\sim 356 \mathrm{~mm})$ while the sensitive area of the module would benefit from an increase in diameter. As a trade-off, the form will slightly diverge from that of a perfect sphere in order to maximize the number of PMTs per module resulting in two 14-inch hemispheres with cylindrical extensions of a few centimeters (see Fig. 1). Given the dimensions of currently available PMT prototypes, 

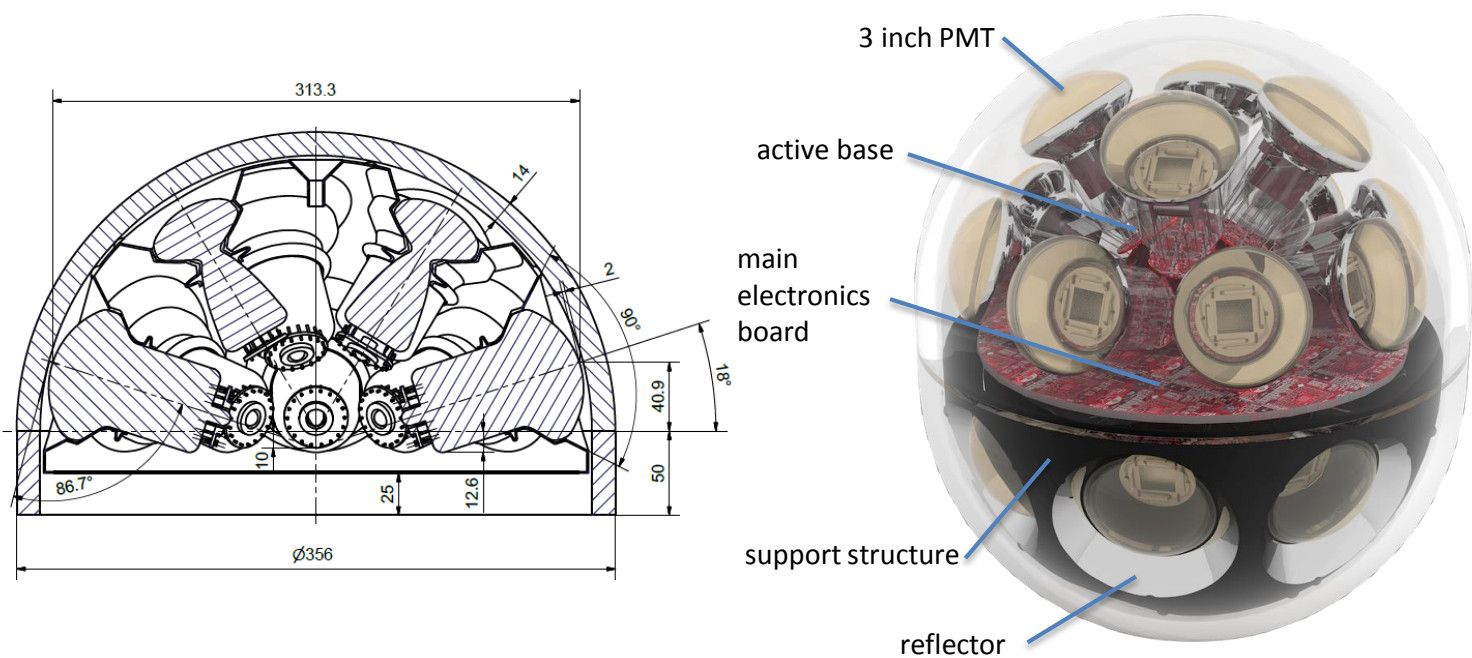

Figure 1: Left: Technical drawing of a mDOM hemisphere. Dimensions are given in mm. Right: Artist's impression of the planned mDOM highlighting the main components. The black holding structure in the bottom hemisphere is omitted in the upper hemisphere to better illustrate the internal components and arrangement.

each hemisphere will be able to house twelve 3-inch PMTs. A crucial requirement on the vessel stability is imposed by the in-ice ambient pressure. IceCube measurements indicate that during refreezing of the water column the vessel has to sustain a pressure of up to $700 \mathrm{bar}(\sim 10,000 \mathrm{psi})$. As corresponding glass housings are currently not commercially available, negotiations with a glass manufacturer (Nautilus $\mathrm{GmbH}$ ) are under way to develop a suitable design for the project. For positioning of the PMTs and auxiliary equipment inside the vessel a supporting structure has been designed. First prototypes were produced from polyamide (type PA 2200 [11]) using laser sintering by a commercial company. For mass production, alternative, more cost-effective production methods are also taken into consideration.

\subsection{Photomultiplier tubes}

At present two companies (Hamamatsu ${ }^{1}$, ET Enterprises ${ }^{2}$ ) provide 3 -inch PMTs $^{3}$ for KM3NeT which are also considered for the use in the IceCube-Gen2 module. These were optimized to fit predefined requirements (see [12]). An excerpt of the achieved performance is listed in Tab. 1. For details on test procedures see [13] and [14]. For prototyping, R12199-02 PMTs by Hamamatsu will be used. However, with its bigger photocathode diameter and shorter tube length, the type D792KFL PMT supplied by ET Enterprises is also well suited for the use in the project. Further

\footnotetext{
${ }_{1}$ http://www.hamamatsu.com/us/en/index.html

$2_{\text {www }}$.et-enterprises.com

${ }^{3}$ The diameters of the originally available 3 -inch PMTs $(\sim 76 \mathrm{~mm})$ have been increased by the manufacturers: to $80 \mathrm{~mm}$ for the R12199-02 by Hamamatsu, and to $86 \mathrm{~mm}$ in the case of D792KFL by ET Enterprises.
} 
candidate PMTs might become available from $\mathrm{HZC}^{4}$ (producing 3-inch PMTs since mid-2013) and MELZ ${ }^{5}$ (prototype presented in April 2015). However, the results of tests performed on initial HZC specimen do not meet with the KM3NeT requirements yet, especially concerning dark rate [15]. Technical modifications of PMT characteristics can be considered once specific requirements are defined for IceCube-Gen2, e.g. lower values are desirable for the dark rate in order to take full advantage of the low background environment in the deep glacial ice.

The utilization of hemispherical PMTs attached to the inner surface of a spherical pressure vessel with lower curvature results in significantly increased mean thickness of gel traversed by the photons arriving from high inclinations which leads to losses due to absorption and reflection. Furthermore, as thick gel layers are costly, in practice the holding structure would be constructed such that it shadows these photons thereby reducing the effective area. Therefore, reflectors in the form of hollow cone rings are used (see Fig. 1, right). These reflector rings, cut and formed from coated aluminum sheets (ALMECO V95100, [16]), redistribute the angular acceptance from high to low inclinations (see Fig. 2) thus increasing sensitivity of the PMTs in the central $\pm 50^{\circ}$ direction by about $30 \%$.

The polarity of the high voltage applied to the PMT is a major decision. A PMT can be operated either with its photocathode at negative high voltage and the anode at ground potential (negative polarity) or, in the case of positive polarity, the cathode is grounded and the anode connected to positive high voltage. Both approaches come with their respective advantages and limitations: While the output of a PMT at negative polarity can be directly fed into read-out electronics, positive polarity demands galvanic separation of the anode (e.g. using a capacitor) which affects the quality of the output. On the other hand, a photocathode at high negative potential promotes increased and unstable dark rates. This effect is more pronounced if objects at ground potential are placed near the PMT, in particular near the photocathode area, like the metal reflectors in case of the mDOM.

Long-term measurements have shown that optical gel covering the photocathode area significantly improves the stability of the dark rate. Whether the gel is sufficiently effective for use of negative high voltage polarity in the $\mathrm{mDOM}$ without losing the advantage of low environmental background in IceCube is currently under investigation. Also under investigation is noise reduction via "HA coating" [17] as proposed by the PMT manufacturer. In this configuration, the outer surface of the PMT outside the photocathode area is coated with conductive paint connected to the photocathode potential and sealed with an insulation layer. Two specimen of such coated PMTs were acquired for performance tests. First measurement show a positive effect on the dark count rate and its stability.

\begin{tabular}{lc|lc}
\hline quantum efficiency (at 404 nm) & $\sim 27 \%$ & supply voltage & $<1400 \mathrm{~V}$ \\
quantum efficiency (at 470 nm) & $\sim 22 \%$ & dark rate $\left(\right.$ at $\left.20^{\circ} \mathbf{C}\right)$ & $0.2-1.5 \mathrm{kHz}$ \\
transit time spread & $F W H M<5 \mathrm{~ns}$ & peak to valley ratio & $>3$ \\
\hline
\end{tabular}

Table 1: Current performance examples of PMTs used for KM3NeT multi-PMT optical modules (selected from [6]). Some requirements have been updated in the course of the development. For original values see [12].

\footnotetext{
$4_{\text {http: }} / /$ /www.hzcphotonics.com/en_index.html

$5_{\text {http: }} / /$ www.melz-feu.ru/
} 


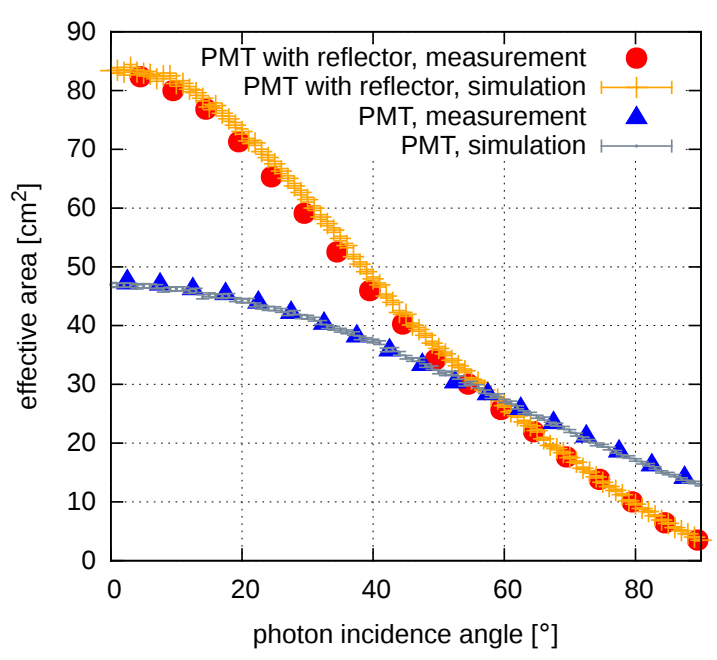

Figure 2: Angular acceptance of a single Hamamatsu R12199-02 PMT in different configurations. The effective area values were calculated normalizing the measured relative signal amplitudes to the geometrical photo-cathode area for perpendicular incidence. The measurement was performed for a bare PMT (triangle) and a reflector equipped PMT mounted on a holding structure (circle). Effective areas from a Geant 4 simulation of the two setups (grey: bare PMT, orange: PMT with reflector) are shown for comparison. Error bars indicate the statistical uncertainties. Simulation is described in section 3.1.

\subsection{Electronics development}

The baseline data acquisition aims at providing information equivalent to the current single PMT technology, enhanced by the pattern of the hit distribution across the PMTs. This leads to a dual digitization approach: On the one hand, the waveform from the sum of the individual PMT signals is digitized. It is unfolded into single photoelectron pulses using a corresponding template waveform. This information can be used to recover the original signal shape. On the other hand, the individual waveforms produced by the PMTs are converted to a time-over-threshold (ToT) step function at the base (via base-borne ASICs or discrete components). The ToT signals are sent to the main logic board, featuring an FPGA and a synchronized clock with a TDC unit, for digitization providing additional time-stamped ToT information for each PMT. Alternative approaches under investigation are extraction of multiple ToTs, local digitization at the base using very fast comparators or the measurement of the pulse maximum during the above-threshold state to discriminate between single high-amplitude signals and multiple low-amplitude pulses arriving in quick succession. Final decisions on the read-out technique will also consider the available data bandwidth of about $1 \mathrm{MBit} / \mathrm{s}$ and a total power budget of less than $4 \mathrm{~W}$ per optical module. A corresponding lowpower active PMT base, including front-end readout electronics, is currently under development.

\section{Simulation efforts}

\subsection{Angular acceptance}

The angular acceptance properties of the module were investigated using a Geant4-based [18] Monte-Carlo simulation of the "effective sensitive area" as a function of photon incident angle and wavelength. The mDOM as modeled in Geant4 includes the glass vessel, a gel infill and reflective cones with their respective optical properties (refractive index, absorption length and scattering length) as well as a totally absorbing PMT holding structure. The PMT model consists of a solid glass bulb containing the photocathode. The rear of the photocathode half-ellipsoid is shielded against photons with a totally absorbing disc. The $\mathrm{mDOM}$ is placed in ice with the appropriate refractive index and infinite absorption and scattering length. In the current setup, photon propaga- 

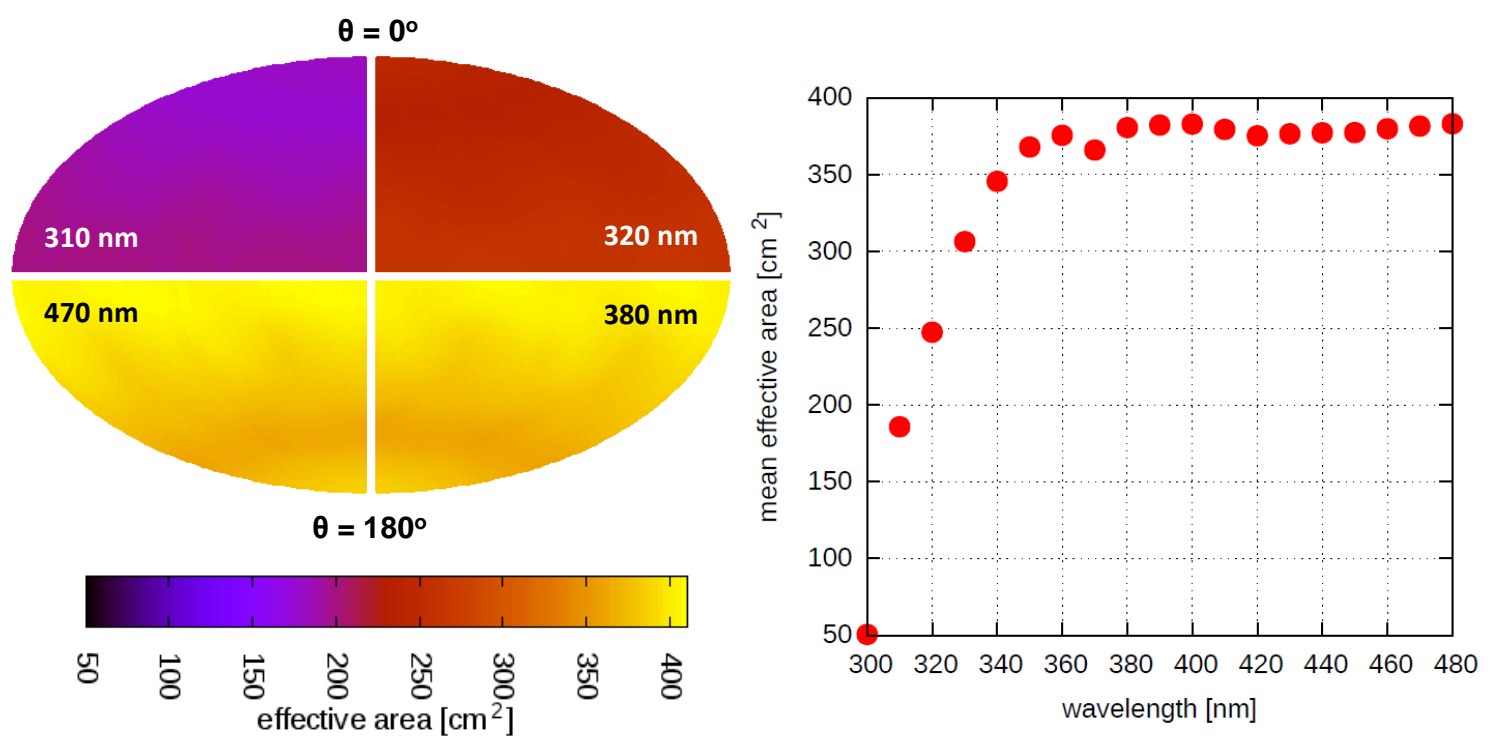

Figure 3: Left: Hammer projection of the $\mathrm{mDOM}$ photon acceptance represented as effective area as a function of direction of the incident photons in zenith angle $(\theta)$ and azimuth $(\phi)$. Values are shown for different original photon wavelengths obtained from a Monte-Carlo simulation based on Geant 4 . The module was oriented as shown in Fig. 1 with vertically down-going photons corresponding to $\theta=0^{\circ}$. Due to the symmetry in the orientation of the PMTs inside the module each of the shown quadrants is representative of the total solid angle for the respective wavelength. For model details see section 3.1 Right: Wavelength dependence of the mean mDOM effective area. Values were obtained averaging solid angle weighted MonteCarlo results over $4 \pi$. Statistical errors are calculated to be smaller than data points.

tion stops at the surface of the photocathode where the photon is registered neglecting the effects of the inner structure of the PMT (such as back-scattering of photons). The impact of the PMT's quantum efficiency is not included. A plane uniformly radiating parallel optical photons in normal direction ( $\lambda$ between 300 and $470 \mathrm{~nm}, N=10^{6}$ per direction) is rotated around the module uniformly sampling the azimuth and zenith angles. The total fraction of photons reaching one of the photocathodes multiplied by the area of the radiator corresponds to the effective sensitive area of the module for a given direction equal to the normal of the plane. At the moment, the simulation was performed for R12199-02 type PMTs only. Results are shown in Fig. 3. The obtained distribution of the effective area features homogeneous coverage of the total solid angle. The dependence on the photon wavelength is dominated by the transmittance of the glass vessel. For means of comparison, a standard IceCube DOM was also simulated using the same approach showing an increase of the average effective area of the module by a factor of 2 . To assess the validity of the model, it was also used to reproduce a lab measurement of the angular acceptance of a single PMT with and without a reflective cone (see Fig. 2). The glass vessel was removed and ice and gel were replaced by air to reflect lab conditions. Simulation and measurement do agree well, with maximum discrepancies below $5 \%$. Reasons for the remaining differences are under investigation. 


\section{2 mDOM detectors}

In order to assess the impact of mDOMs on the performance of future high and low-energy detectors, a corresponding module was implemented in IceTray [19], the standard IceCube simulation framework. In this simulation, charged particles from the neutrino interaction are propagated with Geant 4 which handles the energy loss and the production of Cherenkov radiation. Subsequently, the resulting Cherenkov photons are individually propagated through the ice taking into account the inhomogeneity of the ice properties ( $\mathrm{clsim}^{6}$ was used for photon propagation, ice properties modeling described in [20]). Photons arriving at the surface of an optical module are stored. Propagation inside the module is performed via a simple ray tracing code neglecting refraction. A hit is registered when a photon intersects a PMT represented by a flat thin disc featuring the angular acceptance of a PMT with reflector as measured in the lab. As a further refinement, a signal waveform generator based on measured PMT characteristics will be added.

At present, the simulation is a 40 string detector geometry featuring $22 \mathrm{~m}$ string spacing and a vertical distance of three meters between the mDOMs. The same setup was also simulated using single PMT optical modules. For means of assessing the gain achieved for low-energy (few GeV) neutrino events in such detectors the total number of PMT hits for both detectors was calculated yielding a factor of $\sim 2$ more hits for the $\mathrm{mDOM}$ detector which is in good agreement with the increase of effective area per optical module discussed in section 3.1. Simulations of high energy neutrino detectors and a potential future megaton-scale detector with sensitivity to events at and below the $\mathrm{MeV}$ range for proton decays and supernova neutrinos are in preparation.

\section{Current project status and next steps}

The design of the pressure vessels is being finalized and first specimen are expected to be available in late 2015. 3-inch PMTs from Hamamatsu are available and will be used in mDOM prototyping. A low-temperature (down to $-70^{\circ} \mathrm{C}$ ) test bench has been set up to measure PMT and optical module properties crucial for the use in the deep ice such as the dark rate and background form radioactive decays in the glass in conditions closer to final application. Work on the design of the PMT base incorporating a pre-amplifier and time-over-threshold signal generation is in progress and options for the summation electronics and integration into the IceCube-Gen2 DAQ are being investigated. Simulations currently undertaken address the properties of the module itself as well as the impact of the utilization of mDOMs on the performance of IceCube-Gen2 and a potential future megaton sub-GeV neutrino detector which are expected to benefit from the increased effective area and superior information on the recorded photons.

\section{Acknowledgments}

The low-power active PMT bases for the use in the mDOM project are developed by Andreas Bänisch, Jürgen Röber, Alexander Kölpin, and Stefan Lindner from the Institute for Electronics Engineering of the University of Erlangen-Nuremberg.

${ }^{6}$ C. Kopper, [https://github.com/claudiok/clsim] 


\section{References}

[1] F. Halzen and S. R. Klein, Rev. Sci. Instrum. 81 (2010) 081101 [http://dx.doi.org/10.1063/1.3480478]

[2] ANTARES Collaboration, M. Ageron et al., Nucl. Instrum. \& Meth. A656 (2011) 11 [http://dx.doi.org/10.1016/j.nima.2011.06.103]

[3] BAIKAL Collaboration, I. A. Belolaptikov et al., Astropart. Phys. 7 (1997) 263

[4] IceCube Collaboration, R. Abbasi et al., Nucl. Instrum. \& Meth. A618 (2010) 139 [arXiv: 1002.2442$]$

[5] IceCube Collaboration, R. Abbasi et al., Nucl. Instrum. \& Meth. A601 (2009) 294 [arXiv:0810.4930v2]

[6] KM3NeT Collaboration, R. Bruijn and D. van Eijk, PoS(ICRC2015)1157 these proceedings

[7] KM3NeT Collaboration, S. Adrian-Martinez et al., Eur. Phys. J. 9 C74 (2014) 3056 [arXiv:1405.0839]

[8] D. Hebecker et al., PoS(ICRC2015)1134 these proceedings

[9] IceCube-Gen2 Collaboration, PoS(ICRC2015)1137 these proceedings

[10] IceCube-Gen2 Collaboration, M. G. Aartsen et al., (2014) [arXiv: 1412 . 510 6]

[11] EOS GmbH, Material data sheet - PA 2200, [https://www.shapeways.com/rrstatic/material_docs/mds-strongflex.pdf]

[12] KM3NeT Collaboration, P. Bagley et al., KM3NeT Technical Design Report (2011) [http://www.km3net.org/TDR/TDRKM3NeT.pdf]

[13] S. Aiello et al., Characterization of the 80-mm diameter Hamamatsu PMTs for the KM3NeT project in Proc. of VLVnT 2013, AIP Conf. Proc. 1630 (2014) 118 [http://dx.doi.org/10.1063/1.4902786]

[14] G. Bourlis et al., Characterization of the KM3NeT photomultipliers in the Hellenic Open University in Proc. of VLVnT 2013, AIP Conf. Proc. 1630 (2014) 106 [http://dx.doi.org/10.1063/1.4902783]

[15] R. Bormuth et al., Characterization of the ETEL and HZC 3-inch PMTs for the KM3NeT project in Proc. of VLVnT 2013, AIP Conf. Proc. 1630 (2014) 114 [http://dx.doi.org/10.1063/1.4902785]

[16] Almeco Spa, vega Lighting Applications, [http://www.almecogroup.com/uploads/841-ALMECO_vega_ENG_L101_11_2013_mai1.pdf]

[17] Hamamatsu Photonics, Photomultiplier Tubes: Basics and Applications (2007) [https://www.hamamatsu.com/resources/pdf/etd/PMT_handbook_v3aE.pdf]

[18] S. Agostinelli et al., Nucl. Instrum. \& Meth. A506 (2003) 250

[19] T. R. De Young, IceTray: a software framework for IceCube, in Proc. of CHEP 2004 (2005) p. 463

[20] IceCube Collaboration, M. G. Aartsen et al., Nucl. Instrum. \& Meth. A711 (2013) 73 [arXiv:1301.5361]

[21] IceCube-PINGU Collaboration, M. G. Aartsen et al., (2014) [arXiv: 1401.2046 ] 